\title{
Assessment of Feasible Methods Used by the Health Care Industry for Real Time Location
}

\author{
J Pancham \\ Department of IT, Durban \\ University of Technology \\ Durban, South Africa \\ Email: panchamj@dut.ac.za
}

\author{
Richard Millham \\ Department of IT, Durban \\ University of Technology \\ Durban, South Africa \\ Email: richardm1@dut.ac.za
}

\author{
Simon James Fong \\ Department of Computer and \\ Information Science University of \\ Macau. Macau SAR \\ ccfong@umac.mo
}

\begin{abstract}
This position paper surveys relevant literature in order to evaluate Real Time Location System (RTLS) for the health care sector. The first step is to identify the most common aspects required for a feasible health care implementation. The second step is to utilise these identified criteria to evaluate common RTLS technologies. The most feasible technology matching these criteria will be selected. Because the most feasible technology selected from the evaluation may lack one or more of the most common identified aspects for a healthcare RTLS, enhancements of this technology will be proposed to overcome these limitations.
\end{abstract}

Index Terms-Real time location system, RTLS, RFID, Health Care, Bluetooth low energy networks

\section{INTRODUCTION}

A LTHOUGH the capability to track both people and objects outdoors is achievable without difficulty by the use of Global Positioning (GPS) technology, there is also a need to determine the location of people and objects indoors at real time with a high degree of accuracy. Indoor tracking is not feasible through GPS technology [1], due to the dependence on a link between the GPS device and the positional satellites. Consequently, new methods need to be evaluated for indoor tracking. A number of researchers and practitioners have used RTLS for a number of years to address indoor localization in various sectors. According to the definition by [2], an RTLS is a "combination of hardware and software that is used to continuously determine and provide the real time position of assets and resources equipped with devices designed to operate with the system".

The main focus of this research is on the application of RTLS in health care. Within the health care environment researchers are investigating feasible technologies for patient and asset location. These two applications are similar in all respects except for the method of attachment of the device to the asset or patient. Well-managed implementations of RTLS solutions within the health care sector can deliver tremendous value for patient management [3] and [4]; and improved general health care [5]. In addition other important benefits in using RTLS are asset management for easy location of equipment [4] within a health care environment. The results of a survey of the largest public health facility in state of Florida conducted by [6] showed that real time patient monitoring was one of two priorities followed by loca- tion and tracking of medical equipment. In health care there are various constraints, important ones include prevention of interference with the functioning of any medical equipment [7], low cost in terms of price and low transmission power [8]. Furthermore, the selected hospital which forms the exemplar of the RTLS requirements has additional constraints of maximizing battery life, utilizing small form factor devices that can be worn by patients and be able to be used in a scalable variable patient and space environment. The remainder of the concept paper is organized as follows: methodology, results, discussion and conclusion.

\section{MethoD}

The methodology employed utilized a literature exploration to ascertain the methods currently used in the health care sector for indoor RTLS. The literature resulting from this exploration was examined to assess these methods and / or technologies in terms the limitations posed by both the health care field and the hospital exemplar.

\section{A. Research Questions}

The research questions (RQ) addressed by this study are: RQ1. Based on the literature review and hospital exemplar which set of attributes can be identified for an RTLS in health care?

RQ2. Given these identified attributes what common technologies are most feasible in health care with respect to these attributes?

\section{B. Search Process}

The relevant literature was selected using combinations of several expressions on prominent databases containing scholarly publications such as Google Scholar, Elsevier (SCOPUS) and IEEE [9]. The reason for the choice of these databases is that they were readily accessible to the researchers. Key phrases such as "RTLS in healthcare", "low energy localization in healthcare", "patient real time location systems" were used on these search engines and databases.

\section{Delimitation: Inclusion and exclusion criteria}

Due to the rapid advances in of technology, and also to ensure that the latest technologies were assessed, the authors limited the time span of papers in chapters of periodicals, 
journals, and congress proceedings published predominantly between 2012 and 2017. Possible other constraints include backdated articles within this time span, articles not being available due to indexing issues, and only English language articles being chosen. Despite these constraints, it is understood that this study has attained an acceptable appraisal of the chief RTLS systems. This review is not a comprehensive publication appraisal nor a systematic literature review of all scientific literature of the RTLS field, only the most popular RTLS research technologies are presented. These most popular technologies were selected to give some noteworthy descriptive instances of RTLS published in recent peer reviewed works.

From the literature survey the most appropriate attributes in terms of RTLS and exemplar constraints were identified. The health care sector has other constraints such as electromagnetic interference [8], [7] and scalability [10], but space constraints limited our selection to the most appropriate and the most common attributes. In addition to the exemplar of a hospital survey data of 23 US hospitals [11] was used in the evaluation process.

\section{Analysis}

The chosen technologies were assessed against these identified characteristics. Several technologies such as Ultra wide Band [12], infrared, ultrasonic, standard TV signals, computer vision physical contact [13] for health care RTLS were not considered as these do not closely meet the minimum criteria of the essential characteristics such as reasonable cost.

\section{Results}

\section{A. Identified attributes}

We identified five main attributes viz. cost [14], energy consumption [15], detection range [14], size and accuracy [12]. Another attribute scalability is an important factor for consideration as determined by the exemplar. Due to space constraints other attributes such as security were not included in the study. The main criteria identified were used to compare the different principle technologies, these being RFID, Bluetooth and BLE and Wi-Fi. These are discussed together with their advantages and shortcomings. A number of other technologies such as Ultra wide Band [12], which can be used for localization, they were not considered due to the nature of the environment and its constraints.

\section{B. Technologies}

\section{1) $R F I D$}

Despite the extensive research by academics to assist in designing and improving RFID systems over the number of years of the existence of RFID, there are still remains issues that need to be resolved. An RFID tag consists of two components: an antennae that is used to send and receive data and a chip that stores information about the item being tracked [16]. The RFID tag can be classified into three categories viz. passive, semi-passive and active. Active and semi-passive RFID tags have batteries to power their circuits. These active tag uses its battery power source to broadcast radio waves to a reader, whereas a semi-passive tag relies on and external power source i.e. the reader to supply power for its broadcasting. A passive tag on the other hand is composed of an antenna coil and a silicon chip that includes basic modulation circuitry and non-volatile memory. These passive tags rely entirely on the reader to power the tag which intern send its unique identifier [17].

RFID tags are used in numerous applications such as health care and retail. [16] identified two technical issues with RFID viz. tag collision where readers read multiple tags are the same time and are unable to determine the individual identities involved and reader collision where multiple readers read a tag. These technical issues impact negatively on the accuracy of detection. Other issues identified were privacy and signal interference [7]. However, although privacy is an important aspect to be considered in the health care environment, in this case we do not consider any personal or health related information. Signal interference is considered whilst the main attributes identified at being evaluated.

[4] conducted an extensive review of 215 research articles dealing with RFID applications and issues in healthcare. Their findings revealed that the full benefit of using RFID technology will depend different factors including cost.

An additional problem identified by [16] is that many models based on academic research are not implementable and therefore do not help the practitioner. Even when implemented a number of challenges plague the industry preventing wide scale rollout. The main limitations in the implementation of RFID identified by [6] include technical problems such as distractions by metallic objects and electromagnetic interference in reading tags affecting detection range and accuracy as well as the high cost of infrastructure and tags. Solutions to resolve these challenges come at a high cost limiting their feasibility of wide scale implementation.

[6] analysis reveals that cost is an important barrier in RTLS for locating and monitoring of both patients and assets. This conclusion is in agreement with previous research by [18] who found that cost was also an important barrier for both non-implementers and future implementers of RTLS in particular with RFID.

Although the cost of passive tags is low [13] they require more readers as they need to be detected at a very close range, hence increasing total cost. In comparison to passive RFID tags, active RFID tags are more expensive but the accuracy increases to between 1 and 2 meters [12]. Consequently the accuracy of the detection as well as detection range will be dependent on the type of tag being used. Energy consumption is medium as some batteries can have lifespan ranging from months up to a year. However, the size of the device becomes bulky once the battery and holder is fastened. 


\section{2) Bluetooth}

A popular wireless technology used for exchanging data over short distances is Bluetooth. A number of different techniques such as Received Signal Strength Indication (RSSI), trilateration [19] or finger printing are used to increase accuracy for determining location. Accuracy for location differs at a cost in term of power consumption, size of device, and other factors. A number of different methodologies exist to increase the accuracy, the most popular being the RSSI technique which increases accuracy to 1-2 (meters) [20]. An available improvement of RSSI involves a Kamlan filter which increases Bluetooth accuracy to $0.47 \mathrm{~m}$ but at a cost of increased size (due to larger storage requirements) and increased power consumption [14]. As can be seen these RSSI and Kamlan filter techniques adds to the size form factor for Bluetooth and energy consumption. An example of Bluetooth system is Bluetooth Local Infotainment Point (BLIP) which is a managed network offering access to LAN / WAN via Bluetooth [13].

Bluetooth also has drawbacks in crowded areas due to signal attenuation and interference. Bluetooth can transfer large quantities of data, but consumes battery life quickly and costs a lot more [21]. This gave birth to Bluetooth Low Energy (BLE) suitable to exchange little amounts of data consuming using lower energy at a cheaper cost.

\section{3) $B L E$}

BLE is the power-version of Bluetooth that was built for the Internet of Things (IoT) making it perfect for devices that run for long periods on power sources, such as coin cell batteries or energy-harvesting devices [22]. One of the two systems of this version is Bluetooth low energy which transmits small packets of data whilst consuming significantly less power than the previous version of Bluetooth [15] . A BLE system typically consists of a stationary anchor that detect the tags; a tag; and a location engine to calculate the location of the tag [23]. BLE is an improvement and a later version of Bluetooth (BT) offering several advantages such as smaller form factor, lower cost and extended coverage. [24] in their research recognized that the point-to-point communication of the current BLE nodes has only limited coverage over a short range. They propose using a wireless mesh multi-hop network that has multiple nodes that are capable of communicating with each other to enable routing of packets to extend this limited coverage as a possible solution. This distance can be extended further with the combination of current technologies that are more efficient.

Bluetooth ${ }^{\circledR} 5$ released on 6 December 2016 is a transformative update on previous versions that significantly increases the range, speed and broadcast messaging capacity of Bluetooth applications. This version quadruples range and doubles speed of low energy connections while increasing the capacity of connectionless data broadcasts by eight times [25].

$$
\text { 4) } W i-F i
$$

The concept behind Wi-Fi RTLS is that this technology can utilize the existing Wi-Fi infrastructure to communicate with Wi-Fi tags [17]. In order to use Wi-Fi for RTLS additional Wi-Fi access points will need to be installed for a reasonable detection to achieve real time object or people tracking. Wi-Fi is used widely indoors and provides connectivity for a large number of devices. The basic architecture consists of a Wireless Access Point (WAP) and a Wi-Fi device which contains a Wi-Fi module for connectivity. The cost of devices that connect with the WAP will depend on the functionality provided. However the length of battery life will be very low. RTLS uses a single WAP to detect Wi-Fi tags and users Time Difference of Arrival (TDoA) and RSSI to calculate location [17]. The detection accuracy is approximately 3M [26], [12]. Examples of Wi-Fi solutions using Wi-Fi are Ekahau, Microsoft research radar, AeroScout, Intel Place Lab and Pinpoint 3D [13].

The location accuracy for Wi-Fi can be defined as zone, room or subroom level. Even in some cases Wi-Fi tags will be incorrectly detected in a room and to compound this signals cannot be detected by moving assets because of obstructions between the tag and access points [17].

\section{Evaluation of Technologies}

The work by [23], which investigated the reliable tracking of people acknowledged the problems of multipath fading and shadowing often leading to companies using multiple technologies to eliminate the respective disadvantages. Their results showed that detection was unreliable for boundary conditions especially in penetrable walls whilst results were more reliable when tags were closer to the anchors. Hence they concluded that more work was required to improve accuracy.

[27] in their research recommended that further work is needed to investigate alternative systems with lower power consumption and improved accuracy of localization tracking for the health care sector. They also identified optimizing the size and battery life as challenges facing an optimal RTLS.

Due to the complexity for rating total cost, size of devices and energy consumption a total relative rating was used. The measurements for detection range and accuracy are dependent on the type of tag and hardware used. Therefore measurements will have a wide range and will vary depending on the infrastructure used. The technologies selected are the most appropriate for RTLS within a health care environment. The following are key considerations for the different technologies: cost due to the tight budgets of health care institutions, size because the tag will be worn by patients, energy consumption to ensure long battery life as well as enabling the battery to be as small as possible, accuracy to correctly locate a patient or asset and detection range to minimize infrastructure.

The results of the evaluation of 23 hospitals by [11] are indicated in Table 1. Furthermore, it was noted that at the time of the survey only eleven hospitals had systems that were fully operational. Although this can be attributed to various reasons, one of the claims by the vendors is that the systems failed to deliver the precision of systems promised. Future research is required to ensure that detection accuracy 
claims are consistent. The author further claimed that research projects to improve RTLS are not proven beyond the pilot phases or lab experiments in holistic hospital environments [26].

Table 1 REsults of US hospital Survey

\begin{tabular}{|l|l|l|}
\hline Technology used & No of hospitals & $\begin{array}{l}\text { No of hospitals / } \\
\text { Degree of accuracy }\end{array}$ \\
\hline RFID & 17 & $\begin{array}{l}\text { Low }=7, \\
\text { Medium }=8\end{array}$ \\
\hline Ultrasound & 3 & Medium $=3$ \\
\hline Zigbee & 2 & $\begin{array}{l}\text { Medium }=1, \\
\text { High }=1\end{array}$ \\
\hline IR & 1 & High $=1$ \\
\hline UWB & 1 & Low $=1$ \\
\hline
\end{tabular}

The attributes for RFID depend on the tag type (active or passive) and their related equipment [13], [14]. The cost of Bluetooth and BLE modules are both low whilst the cost of Wi-Fi is medium to high [13]. The cost of Bluetooth and $\mathrm{BLE}$ is lower compared to Wi-Fi [13]. The detection range is between 50 and 100 meters for Bluetooth. The accuracy for $\mathrm{Wi}-\mathrm{Fi}$ is 10 to 20 meters [12] compared to $10 \mathrm{~cm}$ to 10 meters for Bluetooth [13], [14]. BLE on the other hand has an accuracy of $3 \mathrm{~cm}$ to 5 meters [28]. Scalability is generally good for all technologies as noted by [13].

The following legend is used for Table 2 due to space constraints:

\section{EC: Energy Consumption;}

DR: Detection Range;

Cost and Energy Consumption constraints: L-

Low, M-Medium, H-High;

Size: S-Small, M-Medium, L-Large

\section{Discussion}

RTLS in health care will enable efficient location of patients, employees and equipment. Although RTLS have realized benefits in some cases further research is required to reduce the serious technical impediments to its implementation with regards to asset management [11]. After an evaluation of the technologies as per the identified attributes listed in Table 2 together with more recent update to and promises by Bluetooth 5 technology [25], BLE was determined as the most appropriate and feasible technology for the purposes of

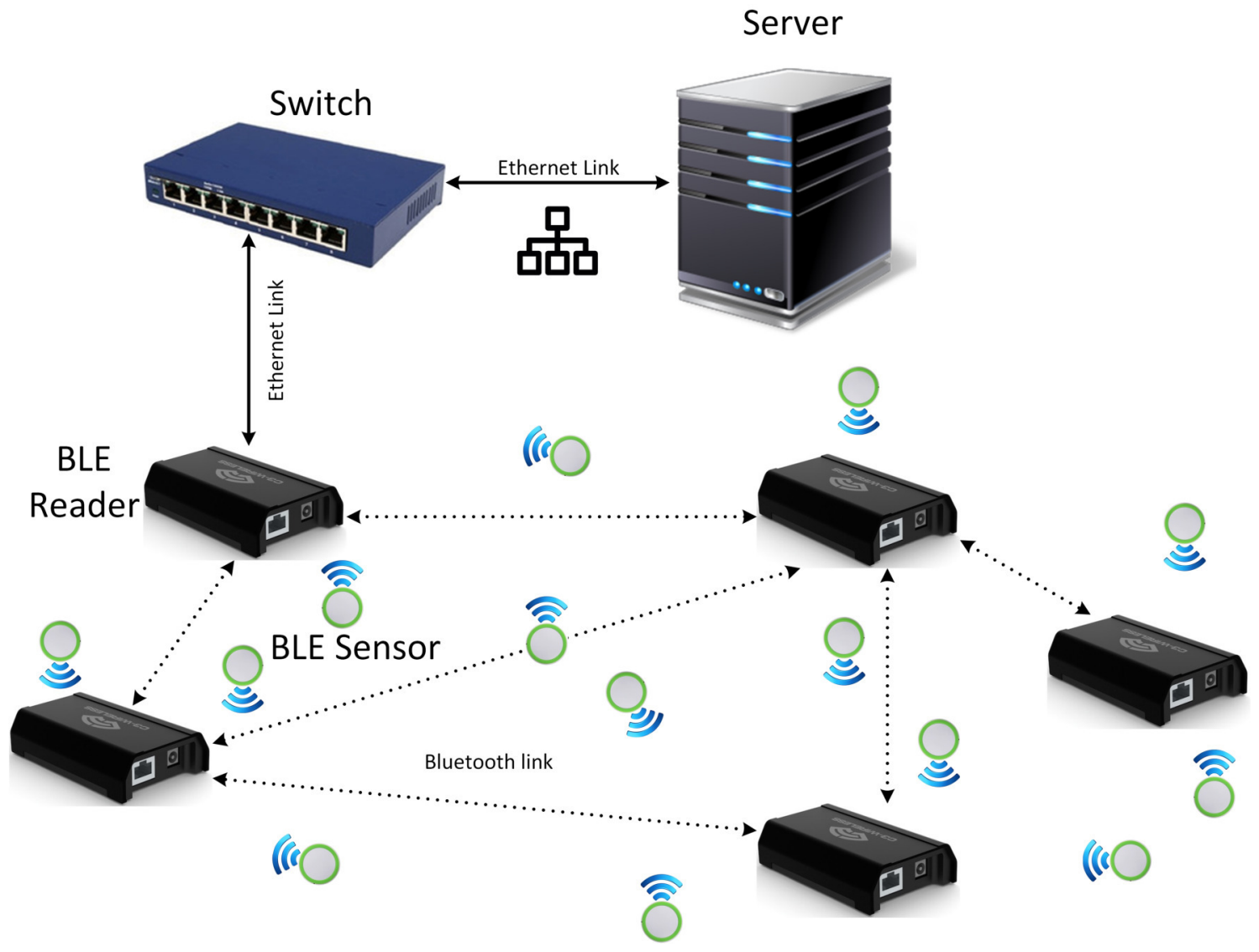

Fig. 1. BLE Network Architecture 
Table 2 Technology Attributes

\begin{tabular}{|l|l|l|l|l|l|l|}
\hline Tech & Cos & E & DR & Size & Accurac & Scalability \\
\hline RFID & H & M & $\begin{array}{l}\text { Depends } \\
\text { on Tag } \\
\text { type }\end{array}$ & L & $\begin{array}{l}\text { Depends } \\
\text { on Tag } \\
\text { type }\end{array}$ & $\begin{array}{l}\text { Good with } \\
\text { minimum } \\
\text { of 2 tags }\end{array}$ \\
\hline BT & M & M & $\begin{array}{l}100 \mathrm{M} \mathrm{for} \\
\text { class 1 }\end{array}$ & S & $\begin{array}{l}10 \mathrm{~cm} \mathrm{to} \\
10 \mathrm{M}\end{array}$ & Good \\
\hline BLE & L & L & $\begin{array}{l}\text { High } \\
(50 \mathrm{M})\end{array}$ & $\mathrm{S}$ & $\begin{array}{l}3 \mathrm{~cm} \mathrm{to} \\
5 \mathrm{M}\end{array}$ & Good \\
\hline Wi- & $\mathrm{M}$ & $\mathrm{H}$ & $\begin{array}{l}\text { up to } \\
200 \mathrm{M}\end{array}$ & $\mathrm{L}$ & $\begin{array}{l}10 \\
20 \mathrm{M} \text { to }\end{array}$ & Good \\
\hline Fi & & &
\end{tabular}

patient and asset tracking for a health care environment. An example of this is that BLE can be configured into a low cost low energy network architecture enabling lower energy consumption [29] and extending the range. The use of BLE devices, with low power consumption will extend battery life thereby reducing maintenance. [30] due to the high volume of patients as well as the size of hospitals especially those in the public sector cost is an important constraint. Therefore these factors were some of the main factors in selecting the most appropriate technology. However BLE suffers from the issues of small detection range and varying degrees of accuracy. Although security is often considered as an important aspect in healthcare, this will need to follow guidelines e.g. (hl7) as set by the health care industry. This will be taken into account during further research.

A combination of BLE tags and BLE readers is required to comply with the requirements for the health care environment.

The BLE reader could be powered by a battery to allow for flexibility and mobility as well as installation of power infrastructure. BLE tags will communicate to BLE readers via blue tooth. The BLE reader will have capability to link to a network switch via an Ethernet cable or a Wi-Fi link. Wi-Fi should prove to be a less disruptive option to be used in the architecture for connectivity back to a server. BLE readers will communicate with each other via Bluetooth and will eventually link back to the switch. A BLE reader can communicate to multiple readers via multiple paths to form a mesh network to cater for redundancy in case of failure of a BLE reader. This high level architecture is depicted in Figure 1. Using this high level architecture, the BLE tags will be connected to a server via the network.

The complexity of the communication and redundancy is housed in the BLE reader so that the BLE tag is less complex reducing its power consumption and cost. BLE readers will be mounted at fixed locations and can therefore have much larger batteries and / or be connected to a power source. The BLE tag will link to the closest BLE readers from which their location can be calculated using a combination of methods. The accuracy of the location required will depend on the usage. Therefore the above configuration allows for a flexible implementation depending on the accuracy required.

After the selection of these technologies which are seen as the most suitable, enhancements involving identifying and implementing appropriate methods to function better in the selected hospital in order to study a live implementation in the practical setting were proposed.

\section{Conclusion}

An accurate and reliable RTLS system within the constraints of the health care environment requires a well-designed architecture. In order to address the challenges identified our approach is to use a combination of BLE tags (sensor) and readers with suitable algorithms to demonstrate the feasibility of an RTLS that mitigates these challenges. A network algorithm will be designed to find the best possible paths in terms of through put, load balancing, and power consumption for communication between BLE tags and BLE readers as well as between the readers. The communication between the different BLE readers in a mesh network will enable coverage of the blind spots not covered by the other readers and will extend the network coverage to increase detection range and improve accuracy as shown in Figure 1. A combination of multiple methods such as triangulation, fingerprinting [31], block chain architecture and repeater tags (tags configured to forward messages) will be used to increase the location accuracy whilst minimizing energy consumption. The availability of multiple communication paths will ensure scalability. By adopting a combination of techniques to improve accuracy and detection range, this might be at the cost of increased complexity, size, and power consumption. This will result in the increase of the cost of the device. Therefore future work will attempt to establish a balance between the primary identified factors

The primary focus of this evaluation was to analyze the relevant literature in the field and to evaluate the technologies for their advantages and disadvantages based on a set of attribute criteria as determined by the literature. Based on this evaluation, the authors selected the most appropriate technology and proposed solutions that addressed their identified shortcomings. Once the pilot sites have been tested the final results will be published in subsequent research articles.

\section{ReFERENCES}

[1] S. Kim, S. Ha, A. Saad, and J. Kim, "Indoor positioning system techniques and security," in e-Technologies and Networks for Development (ICeND), 2015 Forth International Conference on, 2015, pp. 1-4.

International Standards Organization (ISO), "Information technology - Automatic identification and data capture (AIDC) techniques - Harmonized vocabulary - Part 5: Locating systems," vol. ISO/IEC 19762-5, ed. Geneva: ISO, 2007.

[3] N. M. Potisek, R. M. Malone, B. B. Shilliday, T. J. Ives, P. R. Chelminski, D. A. DeWalt, et al., "Use of patient flow analysis to improve patient visit efficiency by decreasing wait time in a primary care-based disease management programs for anticoagulation and chronic pain: a quality improvement study," $B M C$ health services research, vol. 7, p. 8, 2007. 
[4] S. F. Wamba, A. Anand, and L. Carter, "A literature review of RFID-enabled healthcare applications and issues," International Journal of Information Management, vol. 33, pp. 875-891, 2013.

[5] M. Attarha and N. Modiri, "Focusing on the importance and the role of requirement engineering," in the 4th International Conference on Interaction Sciences (ICIS), Busan, Korea, 2011, pp. 181-184.

[6] H. J. Yazici, "An exploratory analysis of hospital perspectives on real time information requirements and perceived benefits of RFID technology for future adoption," International Journal of Information Management, vol. 34, pp. 603-621, 2014.

[7] W. Yao, C.-H. Chu, and Z. Li, "The adoption and implementation of RFID technologies in healthcare: a literature review," Journal of medical systems, vol. 36, pp. 3507-3525, 2012.

[8] H. Alemdar and C. Ersoy, "Wireless sensor networks for healthcare: A survey," Computer Networks, vol. 54, pp. 2688$2710,2010$.

[9] S. Keele, "Guidelines for performing systematic literature reviews in software engineering," in Technical report, Ver. 2.3 EBSE Technical Report. EBSE, ed: sn, 2007.

[10] M. N. K. Boulos and G. Berry, "Real-time locating systems (RTLS) in healthcare: a condensed primer," International journal of health geographics, vol. 11, p. 25, 2012.

[11] J. A. Fisher and T. Monahan, "Evaluation of real-time location systems in their hospital contexts," International journal of medical informatics, vol. 81, pp. 705-712, 2012

[12] Z. Deng, Y. Yu, X. Yuan, N. Wan, and L. Yang, "Situation and development tendency of indoor positioning," China Communications, vol. 10, pp. 42-55, 2013.

[13] G. Deak, K. Curran, and J. Condell, "A survey of active and passive indoor localisation systems," Computer Communications, vol. 35, pp. 1939-1954, 2012.

[14] P. Tsang, C. Wu, W. Ip, G. Ho, and Y. Tse, "A Bluetooth-based Indoor Positioning System: A Simple and Rapid Approach," Annual Journal IIE (HK), vol. 35, pp. 11-26, 2015.

[15] B. Yu, L. Xu, and Y. Li, "Bluetooth Low Energy (BLE) based mobile electrocardiogram monitoring system," in Information and Automation (ICIA), 2012 International Conference on, 2012, pp. 763-767.

[16] X. Zhu, S. K. Mukhopadhyay, and H. Kurata, "A review of RFID technology and its managerial applications in different industries," Journal of Engineering and Technology Management, vol. 29, pp. 152-167, 2012.

[17] B. Wang, M. Toobaei, R. Danskin, T. Ngarmnil, L. Pham, an H. Pham, "Evaluation of RFID and Wi-Fi technologies for RTLS applications in Healthcare Centers," in Technology Management in the IT-Driven Services (PICMET), 2013 Proceedings of PICMET'13:, 2013, pp. 2690-2703

[18] P. M. Reyes, S. Li, and J. K. Visich, "Accessing antecedents and outcomes of RFID implementation in health care," International Journal of Production Economics, vol. 136, pp. 137-150, 2012.
[19] M. D'Aloia, F. Cortone, G. Cice, R. Russo, M. Rizzi, and A. Longo, "Improving energy efficiency in building system using a novel people localization system," in Environmental, Energy, and Structural Monitoring Systems (EESMS), 2016 IEEE Workshop on, 2016, pp. 1-6.

[20] M. Bal, H. Xue, W. Shen, and H. Ghenniwa, "A 3-D indoor location tracking and visualization system based on wireless sensor networks," in Systems Man and Cybernetics (SMC), 2010 IEEE International Conference on, 2010, pp. 1584-1590.

[21] D. Zaim and M. Bellafkih, "Bluetooth Low Energy (BLE) based geomarketing system," in Intelligent Systems: Theories and Applications (SITA), 2016 11th International Conference on, 2016, pp. 1-6.

[22] (2016, 25 May 2017). Bluetooth Low Energy. Available: https://www.bluetooth.com/what-is-bluetooth-technology/howit-works/low-energy

[23] G. Han, G. J. Klinker, D. Ostler, and A. Schneider, "Testing a proximity-based location tracking system with Bluetooth Low Energy tags for future use in the OR," in E-health Networking, Application \& Services (HealthCom), 2015 17th International Conference on, 2015, pp. 17-21.

[24] S. Raza, P. Misra, Z. He, and T. Voigt, "Building the Internet of Things with bluetooth smart," Ad Hoc Networks, 2016.

[25] (2016, 25 May 2017). Bluetooth 5. Available: https://www.bluetooth.com/what-is-bluetooth-technology/howit-works/bluetooth5

[26] A. A. N. Shirehjini, A. Yassine, and S. Shirmohammadi, "Equipment location in hospitals using RFID-based positioning system," IEEE Transactions on information technology in biomedicine, vol. 16, pp. 1058-1069, 2012.

[27] T. Adame, A. Bel, A. Carreras, J. Melià-Seguí, M. Oliver, and R. Pous, "CUIDATS: An RFID-WSN hybrid monitoring system for smart health care environments," Future Generation Computer Systems, 2016.

[28] R. Faragher and R. Harle, "An analysis of the accuracy of bluetooth low energy for indoor positioning applications," in Proceedings of the 27th International Technical Meeting of The Satellite Division of the Institute of Navigation (ION GNSS+ 2014), Tampa, FL, USA, 2014, p. 2.

[29] S. Ahmad, R. Lu, and M. Ziaullah, "Bluetooth an Optimal Solution for Personal Asset Tracking: A Comparison of Bluetooth, RFID and Miscellaneous Anti-lost Traking Technologies," International Journal of u-and e-Service, Science and Technology, vol. 8, pp. 179-188, 2015.

[30] J.-S. Lee, M.-F. Dong, and Y.-H. Sun, "A preliminary study of low power wireless technologies: ZigBee and Bluetooth low energy," in Industrial Electronics and Applications (ICIEA), 2015 IEEE 10th Conference on, 2015, pp. 135-139.

[31] B. Jachimczyk, D. Dziak, and W. J. Kulesza, "Using the Fingerprinting Method to Customize RTLS Based on the AoA Ranging Technique," Sensors, vol. 16, p. 876, 2016. 